\title{
Transmission characteristics of a TEM waveguide for transient signals by the use of a damped sinusoidal
}

\author{
N. Briest ${ }^{1}$, H. Garbe ${ }^{1}$, and S. Potthast ${ }^{2}$ \\ ${ }^{1}$ Institute of Electrical Engeneering and Measurement Technology, Leibniz Universität Hannover, Hannover, Germany \\ ${ }^{2}$ Bundeswehr Research Institute for Protective Technologies, NBC-Protection, Munster, Germany \\ Correspondence to: N. Briest (briest@geml.uni-hannover.de)
}

Received: 15 December 2014 - Revised: 18 May 2015 - Accepted: 04 June 2015 - Published: 3 November 2015

\begin{abstract}
This article broaches the issue of the propagation of transient signals in gigahertz transverse electromagnetic (GTEM) cells. As a representative for transient signals a damped sinusoidal (DS) is used with three different mid-band frequencies. The signal transmission of the DS in the GTEM1250 is qualified and discussed on the basis of the Pearson correlation coefficient (Pcc). The Pcc gives an overview of the signal transmission quality for all measuring points within the testvolume and signal distortions can be identified. A $100 \mathrm{MHz}$ DS is weakly distorted in several measuring points. The Pcc at those points decreases and a signal shape variance can be assumed. Furthermore inhomogeneities of the GTEM1250 caused by the cell door can be identified.
\end{abstract}

\section{Introduction}

The continuously increasing use of electric and electronic equipment requires a save handling. This includes a defined immunity against unintentional and intentional interference. Furthermore the radiated emission of every electric and electronic equipment is subjected to a normative threshold. Therefore it is necessary to measure the radiated susceptibility and emissions of electronic equipment. These measurements has to be performed under far field conditions. In addition it is very important to isolate the equipment under test (EUT) from the electromagnetic environment. Those two criteria are fulfilled by a gigahertz transverse electromagnetic (TEM) waveguide.
In general a TEM waveguide is suitable to transmit transient signals undistorted. Depending on the usable frequency range this is valid only for selected signals. In this paper the damped sinusoidal (DS) is considered. This signal is of ten used in the electromagnetic compatibility (EMC). One example are High-Power Electromagnetic (HPEM) systems, which use this kind of signal. Another example are electric installations, where sinusoidal interferences during a switching operation are possible. These facts show, that it is very important to evaluate the consequences of a sinusoidal interference for an EUT. To estimate the reaction of any EUT to such an interference, an undistorted transmission is an essential characteristic of the used GTEM cell. A qualification of the transmission of the waveguide is realized in this article. The evaluation is implemented with the Pearson correlation coefficient (Pcc).

The article has the following structure: Initially the measurement setup is described in Sect. 2. This part includes the signal generation of the DS, the measurement of the electric field distribution inside the test volume of the GTEM1250 and the calculation of the Pcc. Afterwards the evaluation of the measurement results follows in Sect. 3. The conclusion discusses the transmission behaviour of the DS inside of the GTEM1250 and the DS as a waveguide classifying signal in frequency as well as in time domain (TD).

\section{Measurement setup}

The transmission characteristics of the GTEM1250 are evaluated with a DS, generated with a vector signal generator. A directly modelling of the DS is not possible. Therefore the damped sinusoidal is generated with a sine modelled double exponential function. In Fig. 1 an example of the output volt- 


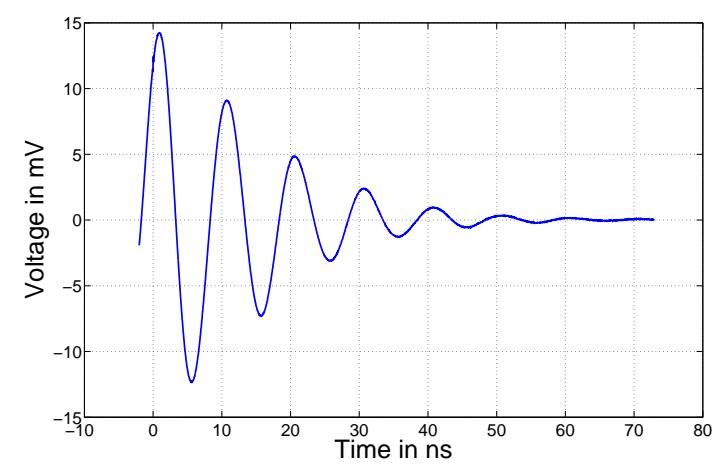

Figure 1. $100 \mathrm{MHz}$ damped sinusoidal (DS).

age of a $100 \mathrm{MHz}$ DS is shown. The voltage output is directly monitored with an oscilloscope.

The maximum output voltage of the signal generator is $1 \mathrm{~V}$. Losses along the cables and the field probe antenna factor require a amplified signal.

The field probe is optoelectronic. Within the test volume of the GTEM1250 the transmission of the $E_{y}$ component of the supplied DS is evaluated. The test volume is sampled in 64 measuring points. According to the manufacturers recommendations (TESEQ, 2014) the measurement points are placed in a height of $0.541 \mathrm{~m}$ above the bottom of the cell. In each direction the measuring points have a distance of $0.13 \mathrm{~m}$ to each other.

To qualify the measured signal within the test volume, a reference is required. This reference is measured at a septum height of $0.485 \mathrm{~m}$, whereby the E-field probe is centred to the septum at a height of $0.24 \mathrm{~m}$. At this reference point the measurement is performed with the identical E-field probe that is used in the test volume. The influence of the probe is the same for both measurements and the losses of cables and distortions of the amplifier do not affect the signal comparison.

Depending on the size and shape of a waveguide, a cutoff frequency exists. Above this cutoff frequency higher order modes can propagate in the test volume (Koch, 1998; Thye, 2012). Due to the higher order modes, the TEM mode does no longer dominate the field distribution. The field distribution of the GTEM cell at the chosen reference point is undistorted. No higher order modes are capable of propagation and the field consists only of a TEM-mode.

A correlation between the reference signal $(x)$ and those measured in the test volume $\left(y_{i}\right)$, is a qualification of the transmission. The Pcc $\rho_{i}$ is calculated (Kölling et al., 2011) (Eq. 1).

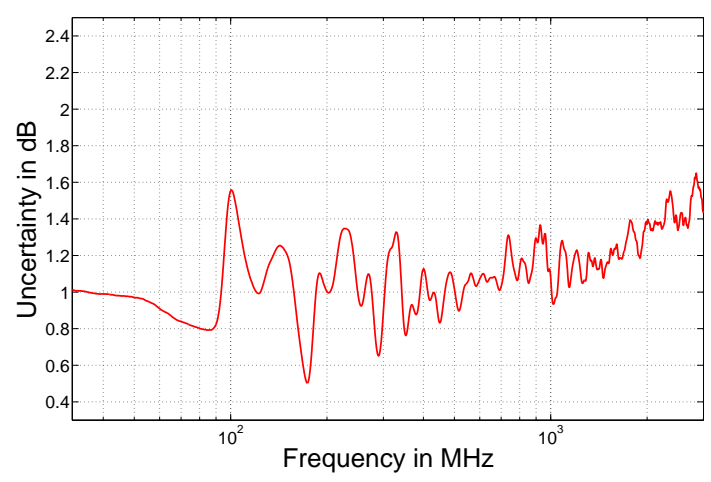

Figure 2. Uncertainty contribution of the field homogeneity within the test volume of the GTEM1250 (Hamann, 2014).

$$
\rho(x, y)=\frac{\frac{1}{N} \sum_{k=1}^{N}(x[k]-\bar{x})(y[k]-\bar{y})}{\sqrt{\frac{1}{N} \sum_{k=1}^{N}(x[k]-\bar{x})^{2}} \cdot \sqrt{\frac{1}{N} \sum_{k=1}^{N}(y[k]-\bar{y})^{2}}}
$$

Discrete frequencies for the supplied DS have to be chosen. According to the manufactures recommendations the GTEM1250 is usable in a frequency range from DC to $18 \mathrm{GHz}$. Hamann and Garbe (2014) investigated this cell in a large frequency band $(30 \mathrm{MHz}-3 \mathrm{GHz})$ and calculated the uncertainty contribution of the field homogeneity, which is depicted in Fig. 2. The uncertainty is constant up to a frequency of $100 \mathrm{MHz}$. Above $100 \mathrm{MHz}$ the uncertainty in the test volume of the GTEM1250 has a high peak. Therefore, a DS with a frequency of $100 \mathrm{MHz}$ is likely to be distorted. Additional characteristic frequencies are 230 and $400 \mathrm{MHz}$. Thus the GTEM1250 has at $230 \mathrm{MHz}$ a worse uncertainty than at a frequency of $400 \mathrm{MHz}$. Those three frequencies show a characteristically uncertainty with a comparable low or high value. The reason for that uncertainty might lead to distortion.

The correlation coefficient for a DS with a frequency of $100 \mathrm{MHz}$ should be much lower than for a DS with a frequency of 230 or $400 \mathrm{MHz}$. One reason is based in the excitation of a higher order mode. Resonances at lower frequencies, e.g. $100 \mathrm{MHz}$, are more severe because of non ideal absorbing structures for this frequency band (Cuming Microwave, 2014). In the following chapter the cell characteristics are discussed and evaluated by the application of a DS to the cell at the three frequencies discussed above.

\section{Evaluation of the transmission characteristics of the GTEM1250 on the basis of the Pcc}

In this chapter the properties of the GTEM1250 are analysed with the correlation coefficient. The correlation between the reference and the signals within the test volume (Fig. 3) of the GTEM cell is visualized in a heat map. 


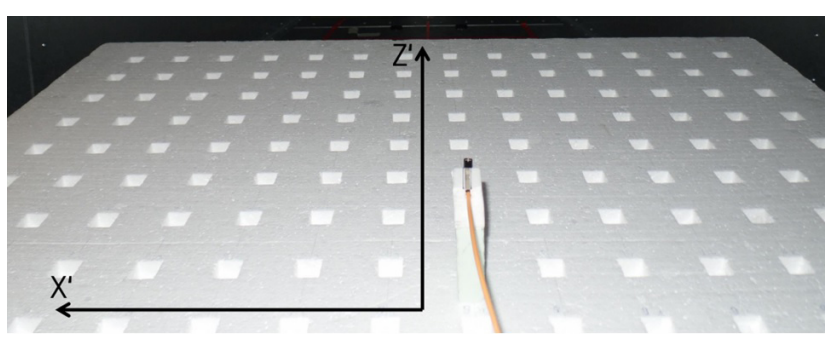

Figure 3. Testvolume of the GTEM1250.

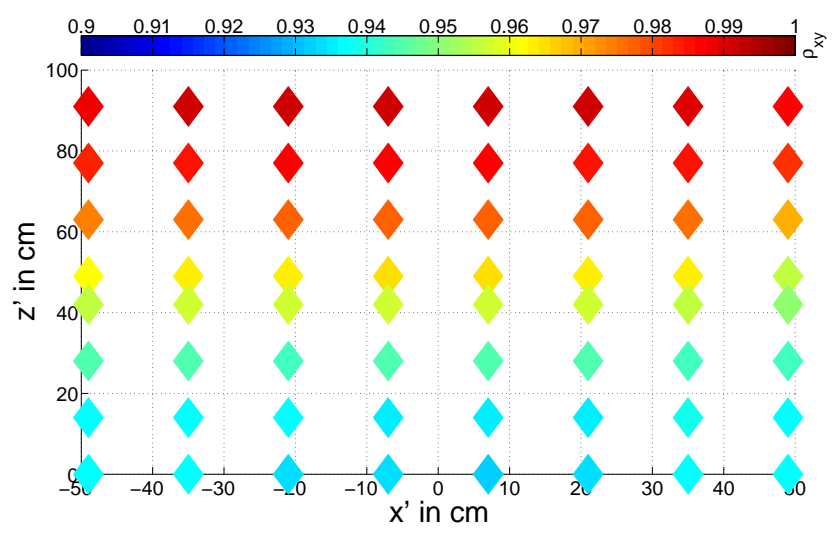

Figure 4. Pcc for a $100 \mathrm{MHz}$ DS within the GTEM1250.

Figure 4 shows the calculated Pcc for a DS with a frequency of $100 \mathrm{MHz}$. The $z^{\prime}$ axis at $x^{\prime}=0 \mathrm{~cm}$ in the shown results is parallel to the middle of the septum. The measuring points along the $z^{\prime}$ axis at $x^{\prime}=-49 \mathrm{~cm}$ and $x^{\prime}=49 \mathrm{~cm}$ are along the edge of the septum. Along the $z^{\prime}$ axis at $x^{\prime}=49 \mathrm{~cm}$ the cell door is located. This geometrical dimensions should be kept in mind during the evaluation of the field distribution using the Pcc. Beginning with a correlation coefficient of $\rho_{x y}=0.99$ at $z^{\prime}=91 \mathrm{~cm}, \rho_{x y}$ decreases continuously in the direction of the rear wall. At the end of the test volume at $z^{\prime}=0 \mathrm{~m}$, the Pcc is $\rho_{x y}=0.93$. Other seen cell characteristics which distort the field distribution are the edges of the septum and the door of the GTEM1250. In comparison with the majority of the Pcc, minimal asymmetries can be seen at these points.

To investigate the signal with a correlation coefficient of $\rho_{x y}=0.93$ more significantly, the signals at the reference and the measuring point are compared in the TD. This is shown in Fig. 5.

The normalisation applied to the signals takes the increasing septum height and the resulting decrease of the electric field strength into account. Due to the normalisation of the signals a comparison becomes more significant. As that reveals non idealities of the waveguide.

In $\mathrm{TD}$, it is possible to show influences of higher order modes more detailed. Reflections can be identified after the first period of the DS, after $6.7 \mathrm{~ns}$, which is equivalent to a reflected wave with a group velocity of $c_{0}=\frac{1}{\sqrt{\mu_{0} \epsilon_{0}}}$ over a

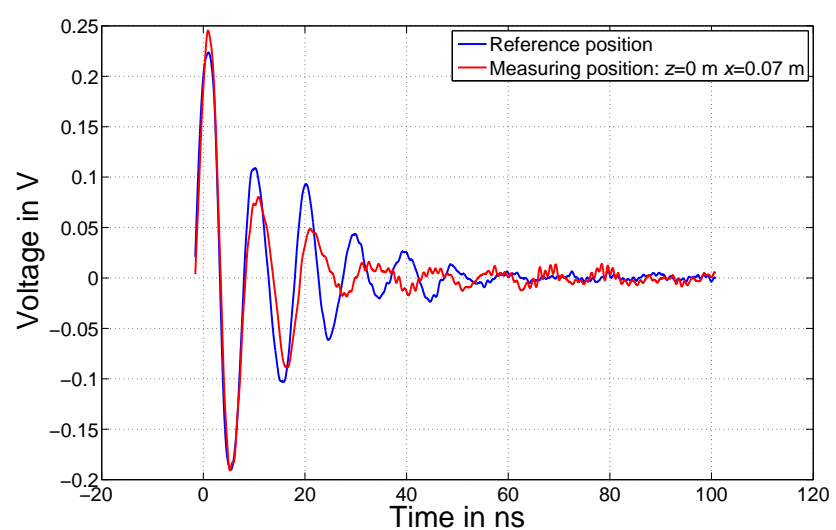

Figure 5. Comparison of the reference signal and the pulse with in the test volume for a $100 \mathrm{MHz} \mathrm{DS}\left(z^{\prime}=0 \mathrm{~m}, x^{\prime}=0.07 \mathrm{~m}\right.$, $\left.\rho_{x y} \approx 0.93\right)$.

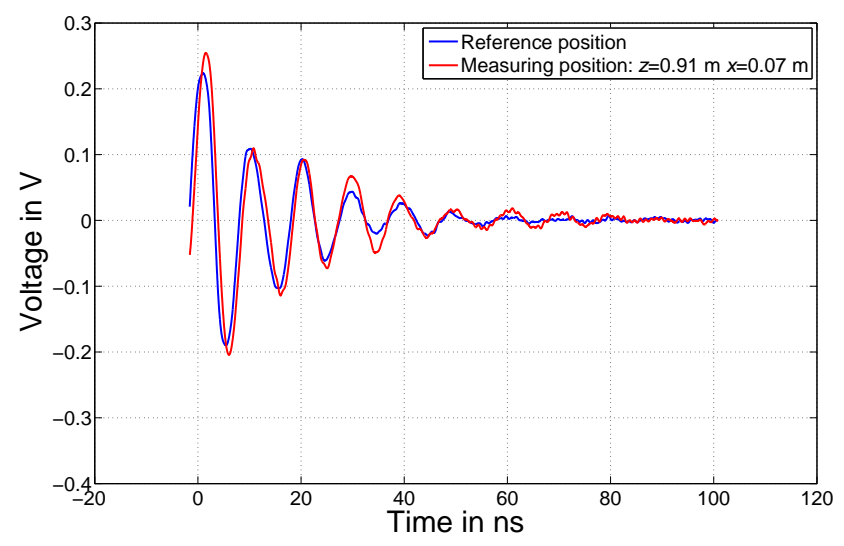

Figure 6. Comparison of the reference signal and the pulse within the test volume for a $100 \mathrm{MHz}$ DS $\left(z^{\prime}=0.91 \mathrm{~m}, x^{\prime}=0.07 \mathrm{~m}\right.$, $\rho_{x y} \approx 0.99$ ).

length of approximately $2 \mathrm{~m}$. This corresponds to twice the distance from the measuring point $z^{\prime}=0 \mathrm{~m}$ (Fig. 4) to the tips of the pyramidal absorbers.

The signals, displayed in Fig. 6, match well for the first period of the DS. After the first period a time delay occurs. In comparison, the measured DS at $z^{\prime}=0.91 \mathrm{~m}$ matches with the reference until the fourth period. With these two examples it can be shown, that the correlation coefficient is a proven quality criterion for a DS. Therefore the differences $\left(\Delta \rho_{x y} \approx 0.06\right)$ of the correlation coefficients is a dimension for the signal deformation.

In addition the GTEM cell characteristics are verified for the DS with center frequencies 230 and $400 \mathrm{MHz}$. According to the uncertainty in Fig. 2, the Pcc for those frequencies should be higher. The Pcc for the test volume of the GTEM1250 at a frequency of $230 \mathrm{MHz}$ is shown in Fig. 7.

The correlation coefficient is constant over the area of the test volume. 62 of the 64 measuring points result in a correlation coefficient between $\rho_{x y}=0.97$ and $\rho_{x y}=0.98$. Below 


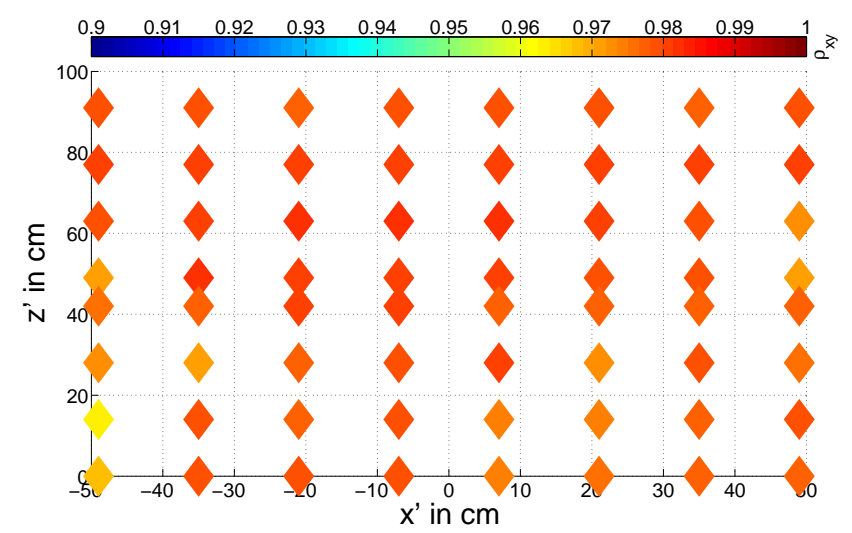

Figure 7. Pcc at a $230 \mathrm{MHz}$ DS within the GTEM1250

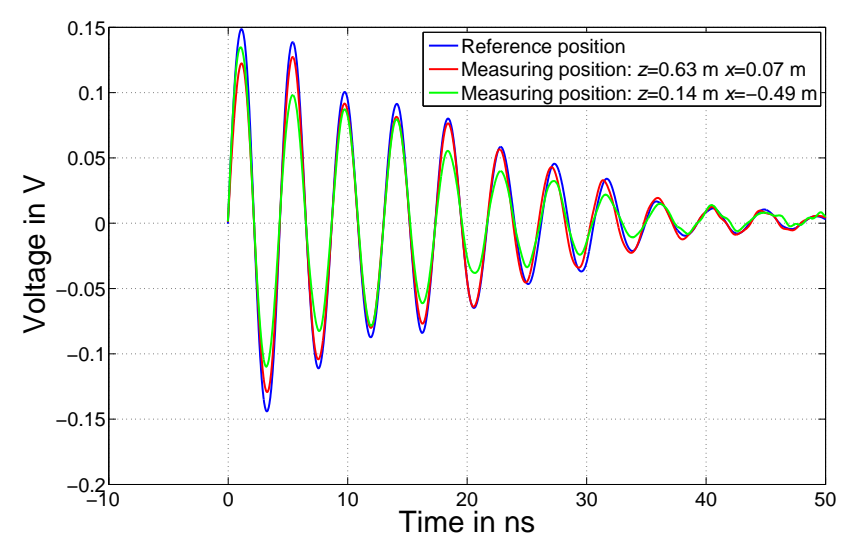

Figure 8. Comparison of the reference signal and the pulses with in the test volume for a $230 \mathrm{MHz} \mathrm{DS}\left(z_{1}^{\prime}=0.63 \mathrm{~m}, x_{1}^{\prime}=0.07 \mathrm{~m}\right.$, $\rho_{x y 1} \approx 0.98 ; z_{2}^{\prime}=0.14 \mathrm{~m}, x_{2}^{\prime}=-0.49 \mathrm{~m}, \rho_{x y 2} \approx 0.96$ ).

the edge of the septum, there are two points where the reference and the signal within the test volume correlate with a coefficient of $\rho_{x y}=0.96$. According to the Pcc a DS at $230 \mathrm{MHz}$ is transmitted much better then at $100 \mathrm{MHz}$. This can be confirmed by results in TD, shown in Fig. 8 .

According to the measurements of Hamann and Garbe (2014) a DS with a center frequency of $400 \mathrm{MHz}$ leads to a smaller uncertainty in comparison to 100 and $230 \mathrm{MHz}$. The effect of this changed uncertainty is evaluated. The resulting correlation coefficients are displayed in Fig. 9.

The overall value of the Pcc of the 64 measurement points is very high. It fluctuates between $\rho_{x y}=0.99$ and $\rho_{x y}=1$ and represents the reference very well. A comparison of the signals with the maximum and minimum correlation coefficient in TD is shown in Fig. 10. Both measured DS match very well with the reference.

According to the results of this work, the GTEM1250 is principally able to transmit transient signals undistorted. This is verified with three DS frequencies and evaluated with the Pcc as well as in TD. In TD a comparison with the measured reference is performed. Frequencies which lead ac-

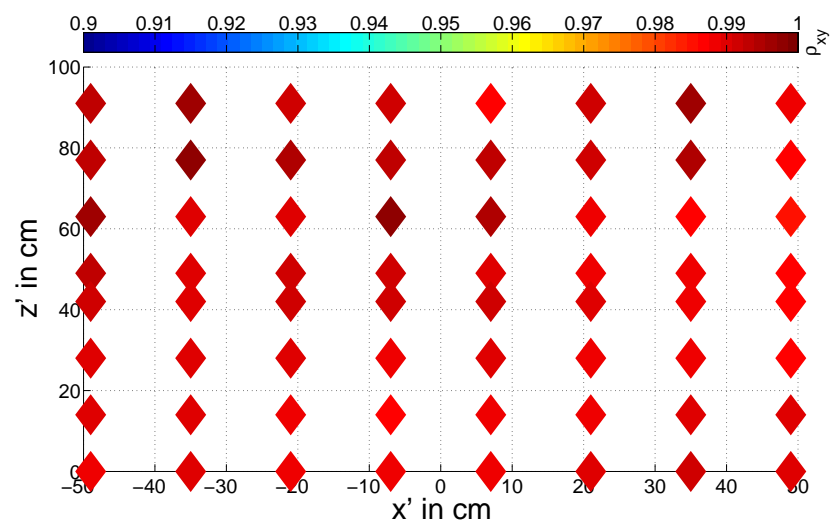

Figure 9. Pcc at a $400 \mathrm{MHz}$ DS within the GTEM1250.

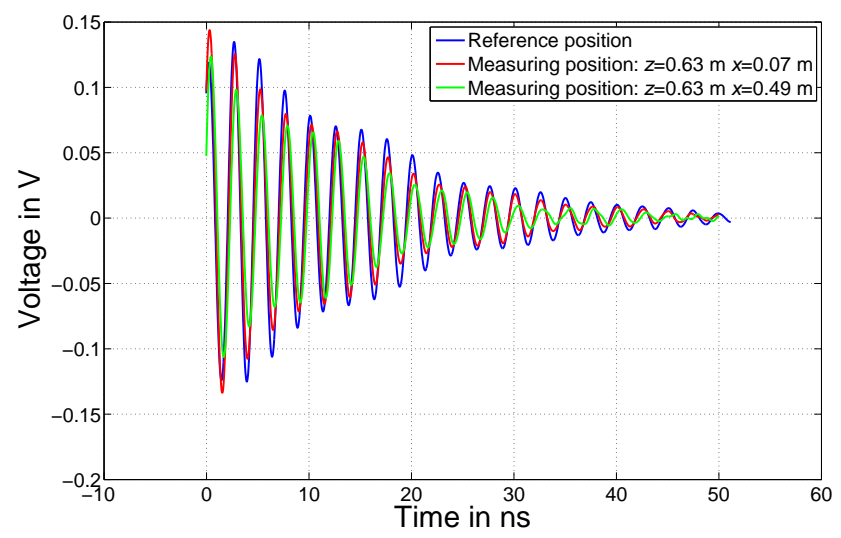

Figure 10. Comparison of the reference signal and the pulses with in the test volume for a $400 \mathrm{MHz}$ DS $\left(z_{1}^{\prime}=0.63 \mathrm{~m}, x_{1}^{\prime}=0.49 \mathrm{~m}\right.$, $\left.\rho_{x y 1} \approx 0.99 ; z_{2}^{\prime}=0.63 \mathrm{~m}, x_{2}^{\prime}=-0.07 \mathrm{~m}, \rho_{x y 2} \approx 1\right)$.

cording Hamann and Garbe (2014) to a higher uncertainty, e.g. $100 \mathrm{MHz}$ ), have a Pcc of $\rho_{x y}=0.93$. The two other DS frequencies, 230 and $400 \mathrm{MHz}$, have a Pcc with a maximum of $\rho_{x y}=1(400 \mathrm{MHz})$. In TD reflections can be observed for the $100 \mathrm{MHz}$ DS after the first period. An inhomogeneous field distribution can be seen. The measured 230 and $400 \mathrm{MHz}$ DS correspond to the reference signal within in complete test volume. The field distribution at these frequencies is much better.

The shown transmission characteristic of the GTEM1250 and the differences for the three discussed frequencies belong to the excitation of higher order modes. For $100 \mathrm{MHz}$ the first higher order mode can be observed and reflections become more dominant. At $250 \mathrm{MHz}$ there are other higher order modes and those are - contrary to the one at $100 \mathrm{MHz}$ - mitigated by the absorbers! 


\section{Conclusions}

The results of this work show that the Pearson correlation coefficient can be used to qualify the waveguide with respect to signal distortion; thus measuring points with a shape invariant signal transmission can be identified. The transient signals used for these measurements were DS waveforms with a center frequency chosen with regard to an uncertainty analysis of former frequency domain measurements. It is shown that resonances causing a large uncertainty in frequency domain result in reflections in time domain and thus in a decrease of the Pearson correlation coefficient. At frequencies with a lower field induced uncertainty dispersion caused by the excitation of higher order modes can be identified as a source slight variations in the Pearson correlation coefficient. With these time domain measurements it can be shown that small variations of the Pearson correlation coefficient identify a deformed signal shape. Although there still is a strong correlation between reference and signal, it is possible to use the Pearson correlation coefficient in order to identify critical signal properties - e.g. a frequency of the DS corresponding to the resonance of a higher order mode. In addition the evaluation of the measurements reveal positions within the test volume where additional investigations might be required as imperfections in the geometry of the waveguide distort the electromagnetic fields.
Acknowledgements. We acknowledge support by Deutsche Forschungsgemeinschaft and Open Access Publishing Fund of Leibniz Universität Hannover.

Edited by: F. Gronwald

Reviewed by: L. O. Fichte, F. Gronwald, and another anonymous referee

\section{References}

Cuming Microwave: C-RAM SCF, http://www.cumingmicrowave. com/pdf/390-Anechoic_Chamber_Mat'1s/390-1_C-RAM_SFC. pdf (last access: June 2015), 2014.

Hamann, D.: Charakterisierung komplexer Systeme in GTEMZellen, PhD Thesis, Sierke Verlag, Hannover, 2014.

Hamann, D. and Garbe, H.: Enhanced Estimates of Field Distributions Uncertainty Contribution for TEM Waveguides, IEEE EMC, Raleigh, 2014.

Koch, M.: Analytische Feldberechnung in TEM-Zellen, PhD Thesis, Shaker Verlag, Hannover, 1998.

Kölling, C., Zamow, D., and Garbe, H.: A Correlation Method to Extend the IEC 61000-4-20 for UWB Measurements, 10th IEEE Int. Symposium on Electromagnetic Compatibility, York, UK, 2011.

TESEQ: Definition of Measurement Points for Field Uniformity, http://www.teseq.de/en/products/downloads/brochure/GTEM_ Cells.pdf (last access: June 2015), 2014.

Thye, H.: Zur transienten Wellenausbreitung in GTEM-Zellen, PhD Thesis, Sierke Verlag, Hannover, 2012. 